\title{
Deep learning and sensor fusion methods for cognitive load gait difference in males and females
}

\section{Document Version}

Final published version

Link to publication record in Manchester Research Explorer

\section{Citation for published version (APA):}

Alharthi, A. S., \& Ozanyan, K. B. (2019). Deep learning and sensor fusion methods for cognitive load gait difference in males and females. 229-237. Paper presented at Intelligent Data Engineering and Automated Learning , Manchester, United Kingdom. https://link.springer.com/chapter/10.1007/978-3-030-33607-3_25

\section{Citing this paper}

Please note that where the full-text provided on Manchester Research Explorer is the Author Accepted Manuscript or Proof version this may differ from the final Published version. If citing, it is advised that you check and use the publisher's definitive version.

\section{General rights}

Copyright and moral rights for the publications made accessible in the Research Explorer are retained by the authors and/or other copyright owners and it is a condition of accessing publications that users recognise and abide by the legal requirements associated with these rights.

\section{Takedown policy}

If you believe that this document breaches copyright please refer to the University of Manchester's Takedown Procedures [http://man.ac.uk/04Y6Bo] or contact uml.scholarlycommunications@manchester.ac.uk providing relevant details, so we can investigate your claim.

\section{OPEN ACCESS}




\title{
Deep Learning and Sensor Fusion Methods for Studying Gait Changes Under Cognitive Load in Males and Females
}

\author{
Abdullah S. Alharthi ${ }^{(凶)}$ and Krikor B. Ozanyan \\ School of Electrical and Electronic Engineering, The University of Manchester, \\ Sackville Street, Manchester M1 3BU, UK \\ \{abdullah.alharthi, k. ozanyan\}@manchester.ac.uk
}

\begin{abstract}
Human gait is the manner of walking in people. It is influenced by weight, age, health condition or the interaction with the surrounding environment. In this work, we study gait changes under cognitive load in healthy males and females, using machine learning methods. A deep learning model with multi-processing pipelining and back propagation techniques, is proposed for cognitive load gait analysis. The IMAGiMAT floor system enabling sensor fusion from plastic optical fiber (POF) elements, is utilized to record gait raw data on spatiotemporal ground reaction force (GRF). A deep parallel Convolutional Neural Network $(\mathrm{CNN})$ is engineered for POF sensors fusion, and gait GRF classification. The Layer-Wise Relevance Propagation (LRP), is applied to reveal which gait events are relevant towards informing the parallel CNN prediction. The CNN differentiates between males and females with $95 \%$ weighted average precision, and cognitive load gait classification with $93 \%$ weighted average precision. These findings present a new hypothesis, whereas larger dataset holds promise for human activity analysis.
\end{abstract}

Keywords: Convolutional Neural Networks (CNN) - Cognitive load gait • Ground Reaction Force (GRF) • Layer-Wise Relevance Propagation (LRP)

\section{Introduction}

In recent years, studies demonstrated that gait is no longer to be considered as an automated motor activity that receives minimal cognitive input [1,2]. Purposeful gait is crucial for individual's aims and environment's burden, to concurrently execute cognitive demanding tasks while walking. Gait cannot be taken as a strict sequential recurrence, with each step and exact copy of the preceding one, due to the unavoidable surrounding environment variability. The progressive adjustment of gait, is considered as a way for humans to minimize the energy cost during the gait cycle, while navigating within often complex surroundings to successfully reach a desired destination $[3,4]$. Humans continuously optimize energy cost in real-time, even if the energy savings while walking are small [5]. Thus, the energy conservation drives changes in gait dynamics, based on cognitive processing of information from the surrounding environment. 
Furthermore, the cognitive function of males and females is a long-discussed issue [6]. Typically, females show preeminence in verbal fluency, perceptual speed, accuracy and fine motor skills; while males outperform females in visuospatial perception processing, mathematical abilities and working memory [7,8]. A recent study on gait kinematics for males and females at self-selected walking speeds, has concluded that the subject's gender can assist in pathology diagnosis, and treatment recommendations in several clinical applications [9].
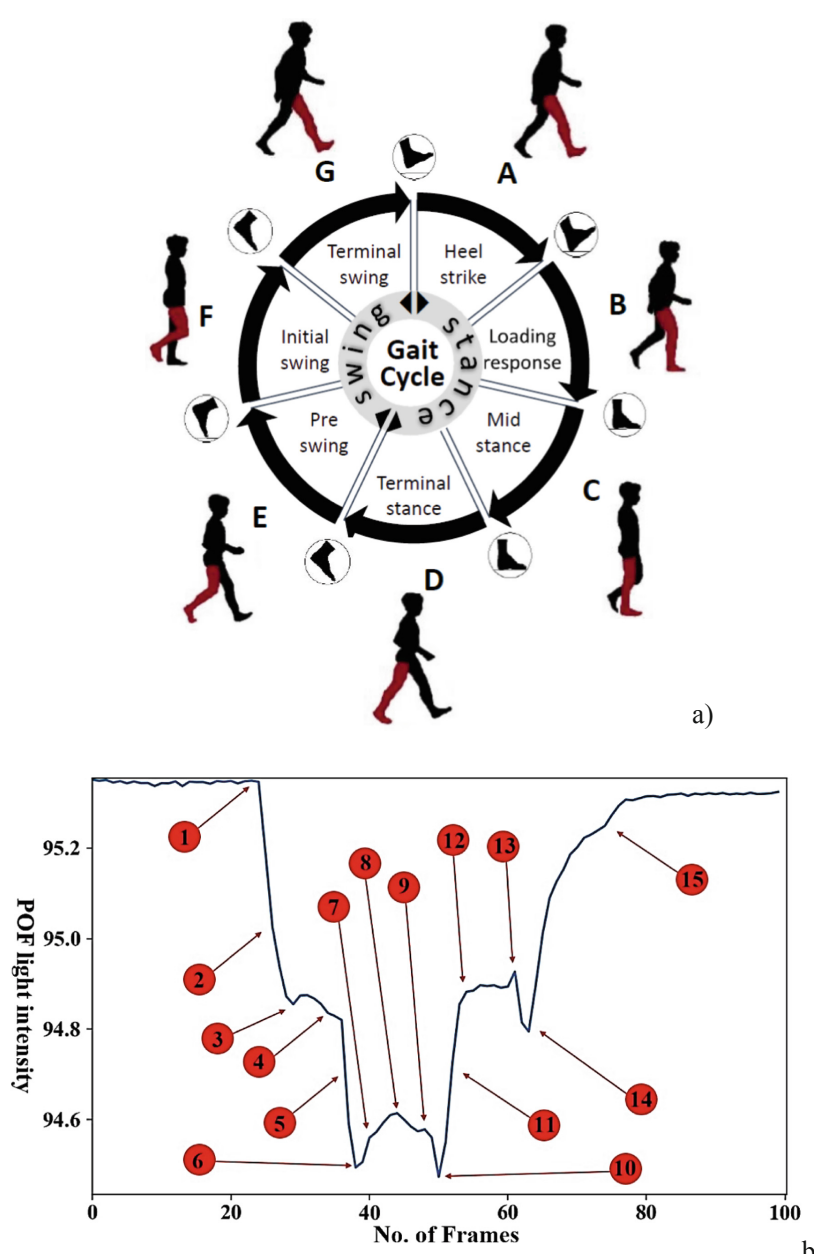

Fig. 1. (a) Gait cycle [12]; (b) Spatial average of gait GRF signals). Gait events recorded by the sensors based on figure (a): 1 - Heel strike, 2 - Foot-flattening, 3 - Single support, 4 - Opposite Heel strike, 5 - Opposite Foot-flattening, 6 - Double support, 7 - Toe-off, 8 - Foot swing, 9 - Heel strike, 10 - Double support, 11 - Toe-off, 12 - Foot swing, 13 - Opposite Heel strike, 14 - Single support, 15 - Toe-off. 
Consistent with this, the gender difference in the response to cognitive load is a focus in this study, based on observing the impact on males and females of a cognitively demanding task while walking. In the longer term, this approach could contribute to improving the quantification of gait decline in studies of cognitive changes (under e.g. comparable stage of Alzheimer's disease) manifested in men and women [10, 11].

The complex gait events and intervals in Fig. 1(a), are typically captured using a well-established sensing technology e.g. cameras to record video sequence of the body motion, wearable sensors to acquire the legs' trajectories and body posture, and sensors under the foot to capture the vertical GRF. Bearing in mind that human walking involves the recurrent contact of the feet with the surface, to deliver a change in the body centerof-mass position. It can be a valuable source of information to study potential differences in the cognitive load impact on males and females. Therefore, floor sensors based on plastic optical fiber (POF) technology [13], is utilized to obtain gait data under the influence of cognitive load. A parallel CNNs network, is engineered to fuse and process the POF sensors signals for cognitive load gait classification. The LRP techniques, is proposed to interpret the CNNs prediction and back-propagate the impact of cognitively demanding tasks. It identifies the event that responsible for influencing the gait cycle.

\section{Methodology}

\section{1 iMAGiMAT System}

The gait cycle depends on the humans' recurrent contact with the ground. Thus several platforms are proposed to capture the footsteps while walking, using sensors fusion to capture walking patterns $[14,15]$. A photonic guided-path tomography sensor head (the iMAGiMAT footstep imaging system [13]), is utilized in this experimental work. An illustration of iMAGiMAT system gait GRF spatial average signal is shown in Fig. 1(b). The spatial average of the POF signals is calculated (See Eq. (1)) to show the temporal activity on the surface of the carpet. As shown further, the processing of the calculated temporal GRF signal, allows interpretation as to which gait event occurred on the carpet surface, using gait cycle descriptors [12].

$$
S A[t]=\frac{1}{116} \sum_{i=1}^{116}\left(R_{i}[t]\right)
$$

$R_{i}$ is the number of sensors in the iMAGiMAT system (116 sensors arranged in three plies, a lengthwise ply with $22 \mathrm{POF}$ at $0^{\circ}$ and two 47 POF arranged diagonally at $60^{\circ}$ and $\left.120^{\circ}\right) . t$ is the number of frames (20 frames per sec).

\subsection{Data Acquisition}

Ethical approval was received through the Manchester University Research Ethics Committee (MUREC) and data acquisition was performed in accordance with the board guidelines. In advance of each data recording trial, each subject answered short question to check over mental state and health conditions. Healthy males and females were invited to walk (while performing cognitive demanding tasks) along the $2 \mathrm{~m}$ length of the 
iMAGiMAT system. Gait spatiotemporal GRF signals were recorded in real time. Five healthy subjects (aged 20 to 31 years, 3 male and 2 females) participated in this experiment. Each subject was asked to walk several steps before and after stepping on the iMAGiMAT system for each experiment, so the captured gait data is unaffected by walking start and stop. 100 frames, each with duration of $5 \mathrm{~s}$, were set for each experiment consistent with a capture frame rate of 20 frames per second. Two dual tasks experiments, as well as normal gait, were acquired for each subject; 20 gait trials for each manner of walking in a single assessment session for male and female, and 10 gait trials for two males and one female. Each manner of walking is identified as a class, as follows:

- Class 0, Normal Gait: walking at normal self-selected gait speed.

- Class 1, gait with serial 7 subtractions: normal gait speed, while at the same time performing serial 7 subtractions (count backwards from 100 by sevens e.g. (93, 86, $79 . .72,65$,) or by count from given random number, stop after completing the task).

- Class 2, gait while texting: normal gait attempted self-selected gait speed while typing in text on a smartphone.

\subsection{Convolutional Neural Network (CNN)}

A parallel CNNs model (see architecture in Fig. 2) is engineered using Keras libraries. The model hyper-parameters are selected based on extensive trial way (hyper parameter optimization). The stacked layers classify subjects' gait under cognitive load alongside with gait identification for males and females. The network fuses the POF sensors signals in the network deep layers, by mapping the signals to a gait class in supervisory training. The model is trained and validated using a batch size of 100 samples for each iteration; 250 echoes are optimal to train the model. The training and validation sizes are set to be $70 \%$ and $10 \%$ respectively, where $20 \%$ is held for testing the model accuracy. An ADAM (A Method for Stochastic Optimization) is used to train the model, where the parallel layer weight and bias shared and updated simultaneously in each iteration. The loss is computed using categorical cross-entropy in every iteration to minimize the network error. To improve the model performance a regularization method is utilized: a dropout with the size of 0.5 after the last MaxPooling layer was flattened, and an additional dropout with the size of 0.2 was added before the output layer. A softmax classifier is placed at the final layer to classify gait GRF signals.

\subsection{Layer-Wise Relevance Propagation (LRP)}

For most data classification cases a nonlinear deep neural network model acts as a black box, since the reasons as to why the models reached such decisions cannot be traced to physical phenomena. LRP [16] is an emerging machine learning method. It is a tool for understanding and interpreting nonlinear deep neural networks decisions. The aim of using LRP in this study is to understand the contribution of individual gait components in the input $\boldsymbol{x}$ ( $\boldsymbol{x}$ : an iMAGiMAT sensor signal at a specific time frame), to the prediction $\boldsymbol{f}(\boldsymbol{x})(\boldsymbol{f}(\boldsymbol{x})$ : gait dual task class) made by the parallel CNN as a classifier $\boldsymbol{f}$. The gait class prediction is redistributed to each intermediate node via backpropagation as far back as 


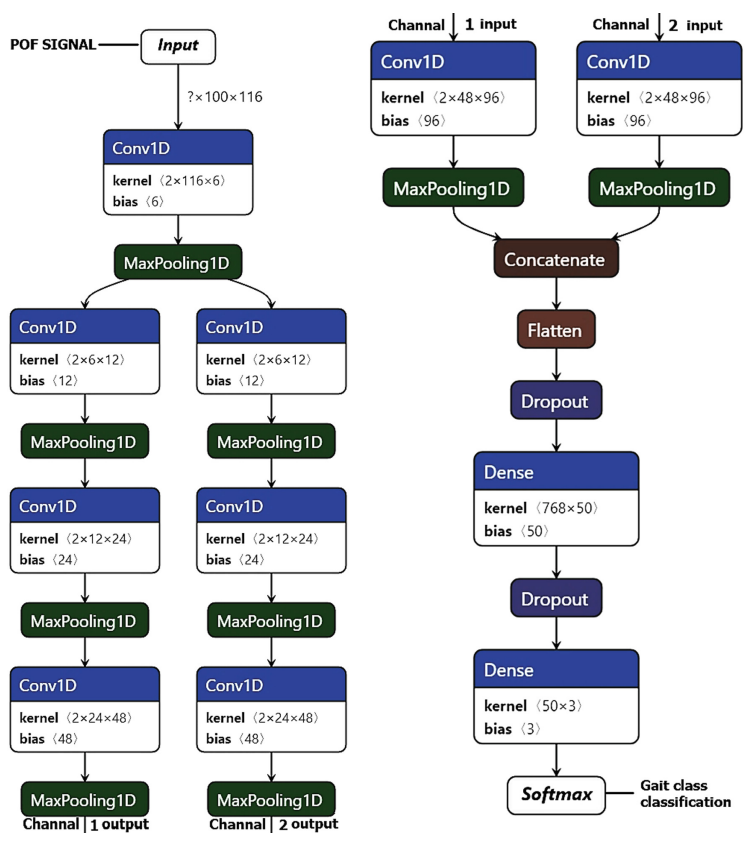

Fig. 2. Parallel CNNs architecture with 2 input and 8 parallel layers, followed by 5 layers.

the input layer. The output of the LRP backpropagation processes, is a heat map with identical size of the original input. The LRP signal highlights spatiotemporal gait pattern relevant for the model prediction. To understand this methodology, we first note that a CNN network consists of multiple computational units of the following form [17]:

$$
(x)_{i}^{s}=l\left(0, \sum_{j} x_{j}^{(s-1)} \omega_{j i}^{(s-1, s)}+b_{i}^{(s+1)}\right)
$$

where $l$ is a non-linear (e.g. ReLU) activation function, $i$ indexes neurons in layer $s, j$ is computed over all neurons joined to neuron $i$ and $\omega_{i j}^{(s-1, s)}, b_{i}^{(s+1)}$ are weight and bias parameters of the $\mathrm{CNN}$, learned during the network training. Many such computational units join to form the entire network. The output $f(x)$ is evaluated in a forward-pass and the parameters are updated by back-propagating using the model error. As shown in [16], using the same network-graph architecture, we can redistribute the total relevance $f(x)$ at the output to input-layer relevance using local redistribution rules ( $\alpha \beta$-rule) [16]:

$$
\boldsymbol{R}_{j}^{(s-1)}=\sum_{i}\left(\alpha \frac{x_{j}^{(s-1)} \omega_{j i}^{(s-1, s)+}}{\sum_{j} x_{j}^{(s-1)} \omega_{j i}^{(s-1, s)+}}-\beta \frac{x_{j}^{(s-1)} \omega_{j i}^{(s-1, s)-}}{\sum_{j} x_{j}^{(s-1)} \omega_{j i}^{(s-1, s)-}}\right) R_{i}
$$

where ()$+$ and ()$-$ denote the positive and negative parts, and the parameters $\alpha$ and $\beta$ are chosen so that $\alpha-\beta=1$ and $\beta \geq 0$ to avoid divisions by zero; here $i$ indexes a feature map for layer $s, j$ computed over all neurons joined to neuron $i$. This rule is 
applied in a backward pass through the network starting at the output layer to produce scores which are called the relevance map. It satisfies the relevance conservation property as per [16]: $\sum_{j} R_{j}^{s}=f(x)$.

\section{Experiments}

The proposed parallel CNN model in Fig. 2, is trained, validated, and tested on $\mathrm{K} \times$ $\mathrm{M} \times \mathrm{N}$ signals; where $\mathrm{K}$ is the number of samples, $\mathrm{M}$ is number of frames, and $\mathrm{N}$ number of sensors. The training and validation size is $168 \times 100 \times 116$, and the testing size is $42 \times 100 \times 116$ GRF signals. The model is trained, validated, and tested three times, to avoid the random weight initialization, and the median result is reported using confusion matrix. Further experimental work is reported in the following sections.

\subsection{Experiment 1}

The aim of this experiment is to categorize gait GRF signals for males and females. The CNN model is trained on identifying the gender of the subject gait, by setting the data labels as a male or female. This two class classification achieved $95 \%$ weighted average precision, and $94 \%$ true positive for males and $96 \%$ for females (on $20 \%$ data unseen by the model) as shown in the confusion matrix in Fig. 3(a).

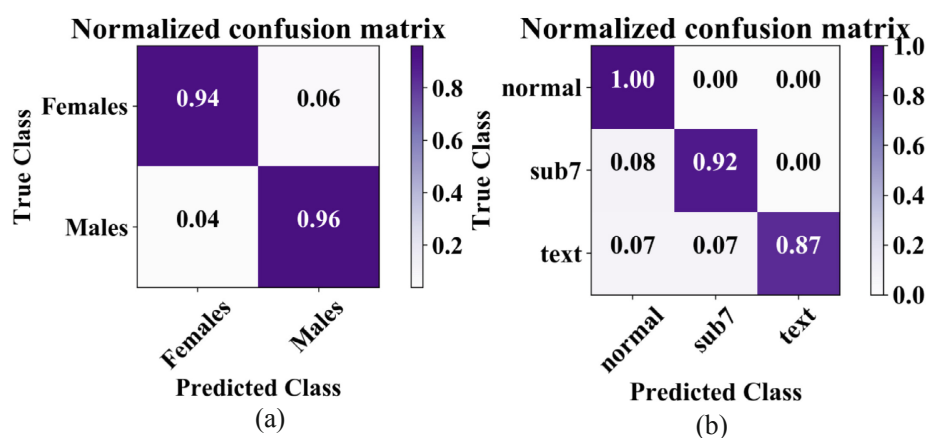

Fig. 3. Gait classification; (a) Males and females classification confusion matrix; (b)

\subsection{Experiment 2}

This experiment focuses on the difference between males and females, in classifying gait changes under cognitive load. The CNN model classified the 5 subjects' gait as normal gait, walking while performing serial 7 subtraction, or walking while texting. The confusion matrix in Fig. 3(b), is illustrating the classification accuracy for cognitive demanding tasks. The model achieved $93 \%$ weighted average precision with $100 \%$ true positives for normal gait, $92 \%$ true positives for gait with subtraction 7 , and $87 \%$ true positives for walking while typing a text on a smartphone. 


\subsection{Experiment 3}

This section addresses cognitive load gait deterioration difference between males and females by deploying the LRP methods of perturbations. The CNN is frozen and transferred using transfer learning methods to perform LRP with $\alpha=2, \beta=1$ using iNNvestigate library [18]. This method shows which gait event contributed positively and negatively in the CNN prediction, (Fig. 4 shows the GRF signals with the corresponding LRP scores for each signal). The model prediction $f(x)$ is back-propagated to gait GRF signals space to outline which gait event (mentioned in Fig. 1(b), using gait events in Fig. 1(a)). The patterns that contributed the most to predict gait class is shown in Fig. 5. The gait spatial average is calculated from the LRP relevance scores, to visually observe the temporal activity on the surface of the iMAGiMAT sensors at a
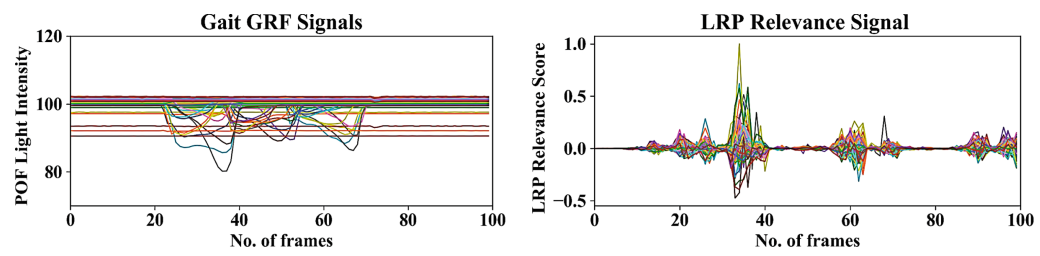

Fig. 4. Raw IMAGiMAT POF signals left, corresponding LRP scores signals right.
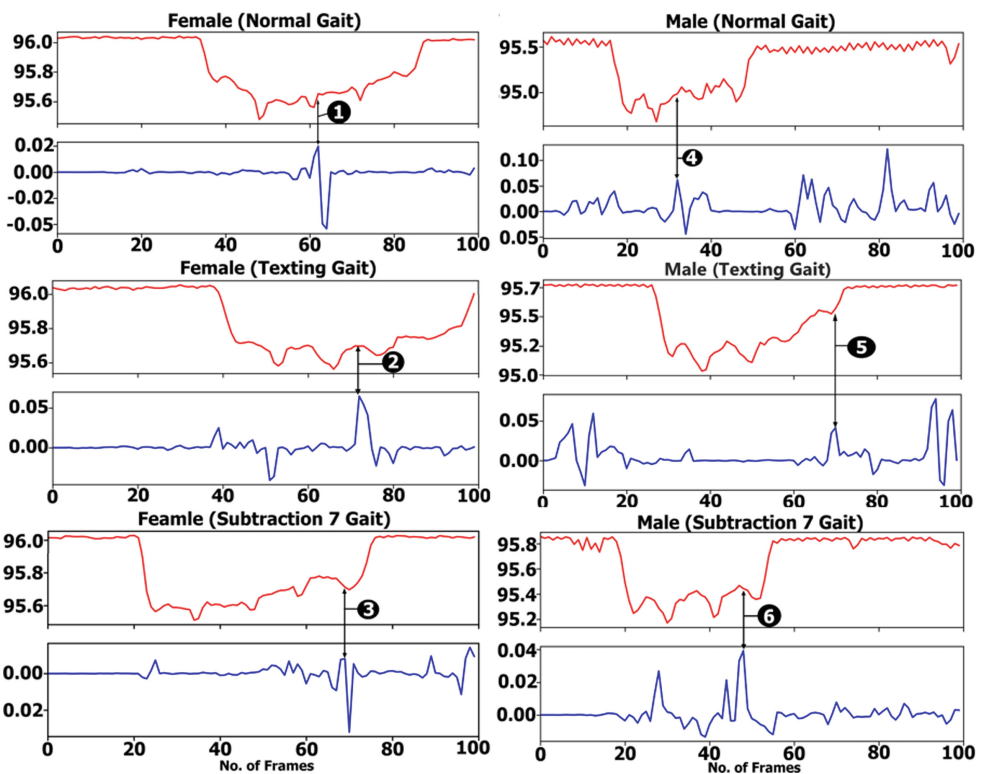

Fig. 5. LRP perturbation applied on males and females data for identifying gait events relevant for the CNN prediction to classify the cognitive load impact on gait. Spatial average of gait GRF signals: red, spatial average for LRP relevance signals over GRF temporal period: blue. Consistent with gait events analysis of GRF spatiotemporal signal in Fig. 1(a) and (b), the cognitive load difference in male and female is as follow: 1 - Toe-off, 2 - Opposite Heel strike, 3 - Foot-flattening, 4 - Foot swing, 5 - Single support, 6 - Opposite Heel strike. (Color figure online) 
specific time. Taken into account that the POF sensors disturbance start when a subject step on the carpet, and the sensed signal will always be initiated by the heal strike followed by the gait events in Fig. 1(a). Further explanation of gait events is in [12].

As observed in Fig. 5, there are positive and negative LRP scores (the positive are numbered from 1 to 6 ). It shows the relevant gait event used towards informing the model's final classification outcome, or the gait event that contributed negatively to reduce the model accuracy. It reveals the difference between gait under cognitive load for males and females, by highlighting the gait events with the highest LRP scores.

\section{Discussion and Conclusion}

We presented a deep learning method to fuse and classify spatiotemporal GRF sensors signals. The gait experimental results for 3 males and 2 females show that gender is distinguishable by $95 \%$. Similar results have been obtained using video sequence [19]. However, in this study, using the GRF of the five subjects, the high classification rate could be resulted from the difference in males and females body weight, posture or muscle strength. Males and females share the same cognitive load, and the classification result is $93 \%$. However, according to the LRP analysis, gait events used for the CNN prediction are different for males and females as shown in Fig. 5. There are high relevance scores when there is no activity on the surface of the carpet. This information is used to inform the model that in some gait classes that the subject speed, was higher than the other classes, or the number of steps on the carpet is less than the other. An explanation of the $\mathrm{CNN}$ classification for the 3 classes is as follows.

- Normal gait: the event in the female gait cycle most influencing the classification is the toe-off, while in males it is the transition between the toe-off and foot swing (see Fig. 5, numbers 1 and 4).

- Walking while texting: the most significant for classification in female gait is the opposite heel strike, while in male gait it is the transition between the opposite heel strike and single support (see Fig. 5, numbers 2 and 5).

- Walking while subtracting in $7 \mathrm{~s}$ : in female gait most affected is the foot-flattening while in males it is the transition between foot-flattening and the opposite heel strike (see Fig. 5, numbers 3 and 6).

The results indicate that males and females do not differ in the accuracy of gait classification under cognitive load; however, LRP analysis suggests that in males the portion of the gait cycle, responsible for the classification of normal gait as well as gait under cognitive load, is slightly lagging behind that of females.

The main limitation of this paper is the small size of the data. Furthermore, the main hypothesis is not well researched in literature, yet it has substantial potential applications in biometrics, healthcare and various other aspects of human activity. The future direction will focus on securing larger data samples of gait GRF for males and females for further in-depth analysis. 


\section{References}

1. Yogev, G., Hausdorff, J.M., Giladi, N.: The role of executive function and attention in gait. Official J. Mov. Disord. Soc. 23(3), 329-342 (2008)

2. Woollacott, M., Shumway-Cook, A.: Attention and the control of posture and gait: a review of an emerging area of research. Gait Posture 16(1), 1-14 (2002)

3. Bertram, J.E.A., Ruina, A.: Multiple walking speed-frequency relations are predicted by constrained optimization. J. Theor. Biol. 209(4), 445-453 (2001)

4. Holt, K.G., Jeng, S.F., Ratcliffe, R., Hamill, J.: Energetic cost and stability during human walking at the preferred stride frequency. J. Motor Behav. 27(2), 164-178 (1995)

5. Selinger, J.C., O'Connor, S.M., Wong, J.D., Donelan, J.M.: Humans can continuously optimize energetic cost during walking. Curr. Biol. 25(18), 2452-2456 (2015)

6. Adenzato, M., Brambilla, M., Manenti, R., et al.: Gender differences in cognitive theory of mind revealed by transcranial direct current stimulation on medial prefrontal cortex. Sci. Rep. 7(41219) (2017). https://doi.org/10.1038/srep41219

7. Sherwin, B.B.: Estrogen and cognitive functioning in women. Endocr. Rev. 24(2), 133-151 (2003)

8. Zaidi, Z.F.: Gender differences in human brain: a review. Open Anat. J. 2, 37-55 (2010)

9. Bruening, D.A., Frimenko, R.E., Goodyear, C.D., Bowden, D.R., Fullenkamp, A.M.: Sex differences in whole body gait kinematics at preferred speeds. Gait Posture 41(2), 540-545 (2015)

10. Laws, K.R., Irvine, K., Gale, T.M.: Sex differences in cognitive impairment in alzheimer's disease. World J. Psychiatry 22(1), 54-65 (2016)

11. McPherson, S., Back, C., Buckwalter, J.G., Cummings, J.L.: Gender-related cognitive deficits in alzheimer's disease. Int. Psychogeriatr. 11(2), 117-122 (1999)

12. Alharthi, A.S., Yunas, S.U., Ozanyan, K.B.: Deep learning for monitoring of human gait: a review. IEEE Sens. J. (2019). https://doi.org/10.1109/JSEN.2019.2928777

13. Cantoral-Ceballos, J., et al.: Intelligent carpet system, based on photonic guided-path tomography, for gait and balance monitoring in home environments. IEEE Sens. J. 15(1), 279-289 (2015)

14. Suutala, J., Fujinami, K., Röning, J.: Gaussian process person identifier based on simple floor sensors. In: Roggen, D., Lombriser, C., Tröster, G., Kortuem, G., Havinga, P. (eds.) EuroSSC 2008. LNCS, vol. 5279, pp. 55-68. Springer, Heidelberg (2008). https://doi.org/ 10.1007/978-3-540-88793-5_5

15. Yun, J., Woo, W., Ryu, J.: User identification using user's walking pattern over the ubiFloorII. In: Hao, Y., et al. (eds.) CIS 2005. LNCS (LNAI), vol. 3801, pp. 949-956. Springer, Heidelberg (2005). https://doi.org/10.1007/11596448_141

16. Montavon, G., Lapuschkin, S., Binder, A., Samek, W., Müller, K.R.: Explaining nonlinear classification decisions with deep taylor decomposition. Pattern Recogn. 65, 211-222 (2017)

17. Bharadhwaj, H.: Layer-wise relevance propagation for explainable deep learning based speech recognition. In: 2018 IEEE International Symposium on Signal Processing and Information Technology (ISSPIT), pp. 168-174 (2018)

18. Maximilian, A., et al.: iNNvestigate neural networks (2018). https://github.com/albermax/ innvestigate

19. Isaac, E.R.H.P., Elias, S., Rajagopalan, S., Easwarakumar, K.S.: Multiview gait-based gender classification through pose-based voting. Pattern Recogn. Lett. 126, 41-50 (2018) 